Check for updates

Cite this: RSC Adv., 2017, 7, 49532

\title{
Monothiatruxene: a new versatile core for functional materials $\uparrow$
}

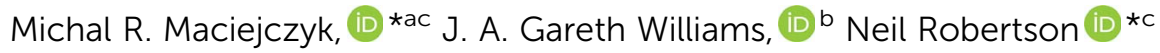 \\ and Marek Pietraszkiewicz ${ }^{a}$
}

A truxene molecule incorporating a sulfur atom at one of the three bridgehead positions has been prepared through an efficient route, together with its oxidized analogue (featuring an $\mathrm{SO}_{2}$ unit) and brominated derivatives. The photophysical, electrochemical and thermal properties of the compounds are presented: the materials show high thermal stability and the ability to form amorphous glasses. This central monothiatruxene core offers multiple routes to further modifications, thereby opening a way to the synthesis of a variety of novel functional materials beyond the parent truxene.

Received 12th July 2017

Accepted 13th October 2017

DOI: $10.1039 / c 7 r a 07671 a$

rsc.li/rsc-advances

synthetic route starting from 1,3,5-tris-(2'-bromophenyl)benzene, as presented in Scheme 1.

The first step, which is a selective substitution of one bromine with methyl sulfide group through lithiation, must be performed at low temperature over at least $1 \mathrm{~h}$ in order to form the most stable lithiated species, ${ }^{18}$ namely the selectively monosubstituted product. To the lithiated substrate, dimethyl disulfide was introduced as a source of sulfur. The pendent sulfur was then oxidized and subsequent ring closure achieved with the aid of acid according to known methods used for the synthesis of dibenzothiophenes. ${ }^{19}$ Lithiation at the remaining bromine sites followed by addition of pentan-3-one gave the dialcohol 4 in a satisfactory yield of $54 \%$; the limit on yield may be caused by enolization of the ketone in the presence of alkyllithium reagents. Condensation and cyclisation of $\mathbf{4}$ works best by using Lewis acids, especially boron trifluoride etherate, giving the expected monothiatruxene product (TrxS) within minutes after the addition of the $\mathrm{BF}_{3}$ reagent, with a high yield of $85 \%$. The monothiatruxene ( $\mathbf{T r x S}$ ) can be easily transformed to the $S, S$-dioxide $\left(\mathrm{TrxSO}_{2}\right)$ upon treatment with hydrogen peroxide in acetic acid, with a yield of $60 \%$ yield.

The final monothiatruxene (TrxS) can be easily modified (Scheme 2) to serve as a versatile platform for functional materials, similarly to the parent truxene molecule. The scheme shows a few examples of derivatization that have been accomplished,
Institute of Physical Chemistry, Polish Academy of Sciences, Kasprzaka 44/52, Warsaw, 01-224, Poland

${ }^{b}$ Department of Chemistry, Durham University, South Road, Durham DH1 3LE, UK 'EaStCHEM School of Chemistry, University of Edinburgh King's Buildings, Edinburgh EH9 3FJ, UK. E-mail: m.maciejczyk@ed.ac.uk; neil.robertson@ed.ac.uk

$\dagger$ Electronic supplementary information (ESI) available: Experimental procedures and ${ }^{1} \mathrm{H},{ }^{13} \mathrm{C}, 2 \mathrm{D}$ NMR and MS data for all new compounds. UV-VIS spectra, SWV traces, XRD patterns, DSC and TGA scans for compounds TrxS and $\mathbf{T r X S O}_{2}$. See DOI: $10.1039 / \mathrm{c} 7 \mathrm{ra} 07671 \mathrm{a}$

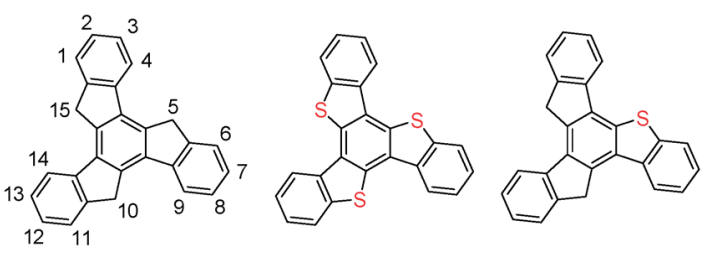

Fig. 1 Structures of truxene, trithiatruxene and monothiatruxene. 

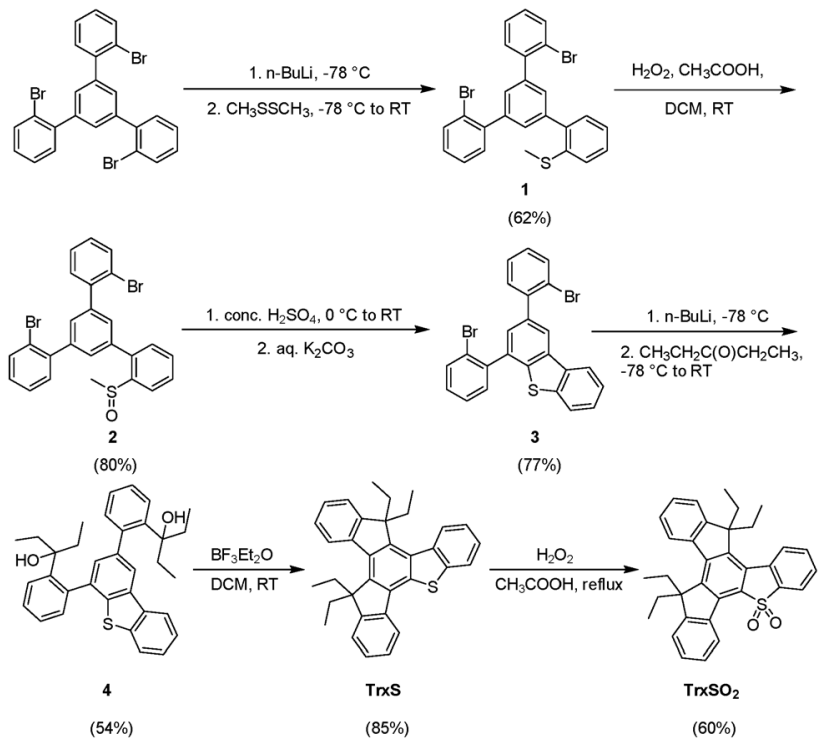

Scheme 1 Synthetic route to monothiatruxene.
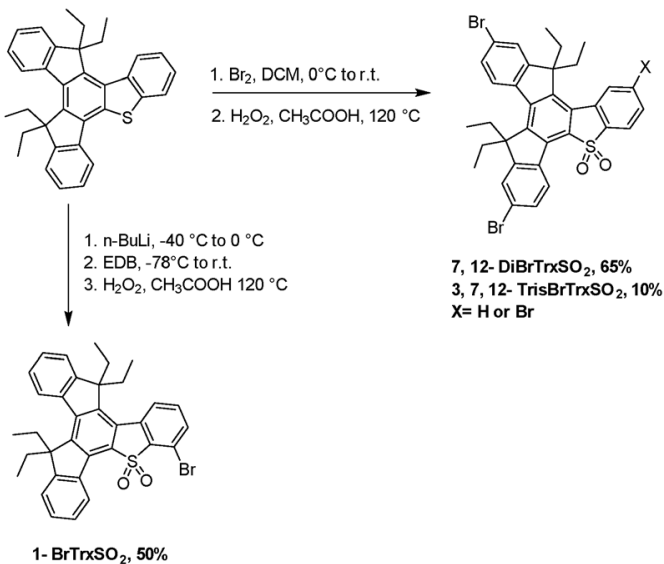

7, 12- DiBrTrxSO, $65 \%$ $3,7,12-$ TrisBrTrxSO $_{2}, 10 \%$ $\mathrm{X}=\mathrm{H}$ or $\mathrm{Br}$

Scheme 2 Derivatization of monothiatruxene.

involving introduction of bromine atoms into different positions, and sulfur oxidation.

Selective bromination of the aromatic ring adjacent to the sulfur atom (position 1) has been achieved by ortho-lithiation and subsequent treatment with 1,2-dibromoethane. Di- and tribrominated derivatives were obtained with the use of simple bromination method with the use of bromine in dichloromethane. Non-oxidized intermediates, namely 1-BrTrxS, 7,12DiBrTrxS and 3,7,12-TrisBrTrxS, were directly subjected to oxidation with hydrogen peroxide. The tribrominated product appeared as a side product of the reaction aiming for the dibromo derivative therefore the final yield is only $10 \%$. Where the aimed 7,12-DiBrTrxSO ${ }_{2}$ has been obtained in $65 \%$ yield. The 1-BrTrxSO $\mathrm{S}_{2}$ was obtained in $50 \%$ yield, after three steps; ortholithiation, reaction with ethylene dibromide and oxidation with hydrogen peroxide. All obtained materials possess good solubility in most common organic solvents even though only ethyl chains were utilized. Detailed reaction descriptions can be found in the ESI. $\dagger$ All the materials were fully characterized by ${ }^{1} \mathrm{H},{ }^{13} \mathrm{C}$ and $2 \mathrm{D}$ NMR spectroscopy and mass spectrometry. Final materials were also characterized by elemental analysis.

The UV-VIS absorption and fluorescence spectra of TrxS and $\mathrm{TrxSO}_{2}$ in dilute dichloromethane solution are presented in Fig. 2. The corresponding spectra in cyclohexane and acetonitrile (less and more polar solvents than $\mathrm{CH}_{2} \mathrm{Cl}_{2}$ respectively) were almost identical, showing no significant solvatochromism (Fig. S1†). Both compounds absorb light only in the UV region with $\lambda_{\max } 288 \mathrm{~nm}\left(\varepsilon=54800 \mathrm{~cm}^{-1} \mathrm{M}^{-1}\right)$ and $285 \mathrm{~nm}(\varepsilon=$ $35000 \mathrm{~cm}^{-1} \mathrm{M}^{-1}$ ) for TrxS and $\mathbf{T r x S O}_{2}$, respectively, with somewhat weaker absorption peaks at longer wavelength: 350 and $366 \mathrm{~nm}$ for TrxS and 357 and $374 \mathrm{~nm}$ for $\mathbf{T r x S O}_{2}$. The emission bands appear at only slightly lower energy than the lowest-energy absorption bands: the Stokes shifts are only $550 \mathrm{~cm}^{-1}$ for TrxS and $940 \mathrm{~cm}^{-1}$ for $\mathbf{T r x S O}_{2}$. This contrasts with unsubstituted hexaalkyltruxene, which has a large Stokes shift of $4600 \mathrm{~cm}^{-1}$. Clearly, $C_{3 \mathrm{~h}}$ symmetry-forbidden transitions in truxene ${ }^{20}$ become allowed upon introduction of the sulfur atom to the core, accounting for the small Stokes shifts. In Fig. S2, $\dagger$ the mirror image relationship between the absorption and emission bands can be observed for both materials. Oxidation of the sulfur atom leads to a less structured spectral profile and red-shifting of the emission maximum: a red-shift of $830 \mathrm{~cm}^{-1}$ in $\mathbf{T r x S O}_{2}$ compared to $\mathbf{T r x S}\left(580 \mathrm{~cm}^{-1}\right.$ in the lowest energy absorption band). The fluorescence quantum yield ( $\left.\Phi_{\mathrm{fl}}\right)$ of $\mathbf{T r x S}$ is $\mathbf{0 . 0 5}$, while oxidation to $\mathbf{T r x S O}_{2}$ increases this value to 0.14 . Table 1 summarizes the photophysical and thermal data.

The oxidation and reduction potentials were obtained from square-wave voltammetry (SWV) experiments using ferrocene as an internal standard (Fig. S3†). Only one oxidation peak of TrxS was recorded at $0.86 \mathrm{~V}$ and no reduction. In contrast, $\mathbf{T r x S O}_{2}$ showed only one reduction peak at $-2.21 \mathrm{~V}$ without oxidation under the same conditions. Both oxidation of TrxS and reduction of $\mathbf{T r x S O}_{2}$ remain unchanged with the scan direction (Fig. S4 $\dagger$ ). The cyclic voltammetry measurement (Fig. 3) at five different scan rates showed that TrxS can be stably and reversibly oxidized (Fig. 3, S5 and Table S1 $\dagger$ ). The cathodic/anodic current ratio is about 1 , proving the stability of the oxidized species.

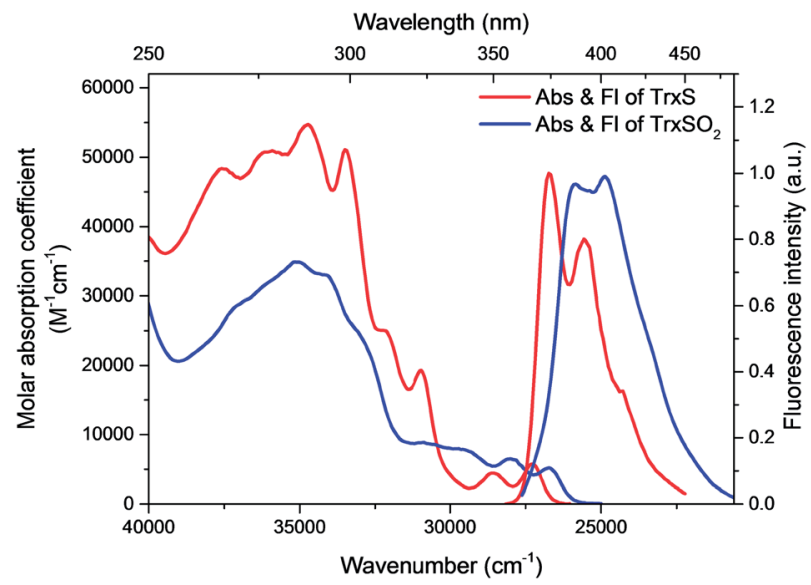

Fig. 2 Absorption and fluorescence spectra of TrxS and $\mathrm{TrxSO}_{2}$ in dichloromethane solution. 
Table 1 Electrochemical, photophysical and thermal properties

\begin{tabular}{|c|c|c|c|c|c|c|c|c|c|c|c|}
\hline & $\lambda_{\mathrm{abs}}{ }^{a}, \mathrm{~nm}$ & $\begin{array}{l}\varepsilon^{a} \times 10^{3} \\
\mathrm{~cm}^{-1} \mathrm{M}^{-1}\end{array}$ & $\lambda_{\mathrm{fl}}{ }^{a}, \mathrm{~nm}$ & $\begin{array}{l}\text { Stokes shift } \\
\mathrm{cm}^{-1}\end{array}$ & $E_{\text {gap }}^{a, b}, \mathrm{eV}$ & $\Phi_{\mathrm{fl}}{ }^{a, c}$ & $E_{\mathrm{Ox}}{ }^{a}, \mathrm{~V}$ & $E_{\text {red }}{ }^{a}, \mathrm{~V}$ & $T_{\mathrm{g}}^{d},{ }^{\circ} \mathrm{C}$ & $T_{\mathrm{m}}{ }^{d},{ }^{\circ} \mathrm{C}$ & $T_{\mathrm{d}}{ }^{e},{ }^{\circ} \mathrm{C}$ \\
\hline TrxS & $\begin{array}{l}288,299 \\
350,366\end{array}$ & $\begin{array}{l}54.8,51.1 \\
4.5,5.7\end{array}$ & 374,391 & 550 & 3.38 & 0.05 & +0.86 & - & 84 & 208,214 & 326 \\
\hline $\mathrm{TrXSO}_{2}$ & $285,357,374$ & $35,6.5,5.2$ & 387,400 & 940 & 3.42 & 0.14 & - & -2.21 & 119 & 256 & 357 \\
\hline
\end{tabular}

${ }^{a}$ In dichloromethane solution. ${ }^{b}$ Optical gap, from intersection of Abs and $\mathrm{Pl}^{c}{ }^{c}$ In reference to qunine sulfate in $0.1 \mathrm{~N} \mathrm{H}_{2} \mathrm{SO}_{4}(\Phi=0.51) .{ }^{d}$ Glass transition temperature $\left(T_{\mathrm{g}}\right)$ and melting point $\left(T_{\mathrm{m}}\right)$ by DSC. ${ }^{e}$ Decomposition temperature $\left(T_{\mathrm{d}}\right)$ by TGA.

Therefore, it may concluded that this redox couple is reversible. On the other hand, the reversibility of the reduction of the $\mathrm{TrxSO}_{2}$ could not be evaluated, as can be seen in the Fig. 3 , since the process is close to the solvent window and both peaks could not be distinguished clearly.

The differential scanning calorimetry (DSC) showed that both materials are of crystalline nature with melting points of $198^{\circ} \mathrm{C}$ and $214{ }^{\circ} \mathrm{C}$ for $\mathbf{T r x S}$ and $256{ }^{\circ} \mathrm{C}$ for $\mathbf{T r x S O}_{2}$ (see Fig. S6 and S7†). After melting the material and heating it again, TrxS showed a glass transition temperature of $84.5^{\circ} \mathrm{C}$ and cold crystallization at $180.6^{\circ} \mathrm{C}$ along with again two melting points at $207.8^{\circ} \mathrm{C}$ and
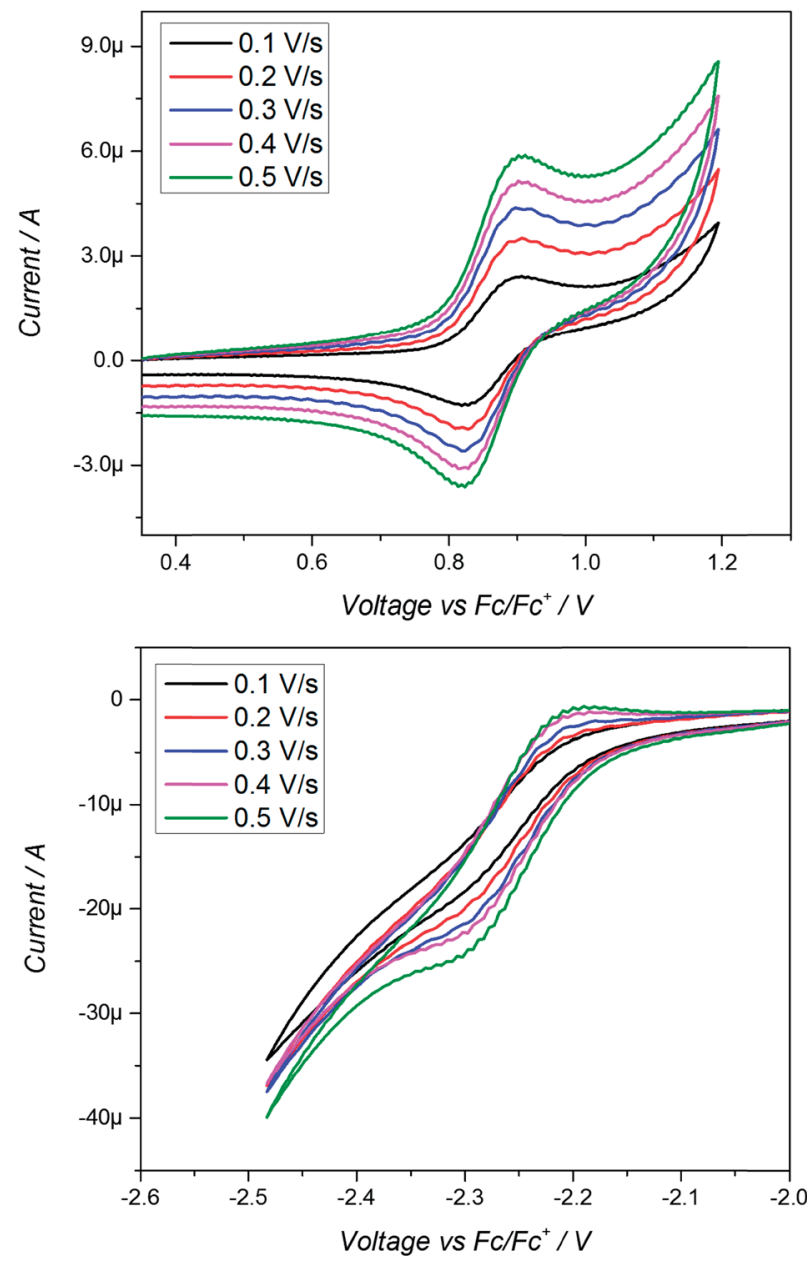

Fig. 3 Cyclic voltammetries of TrxS (top) and $\mathrm{TrxSO}_{2}$ (bottom) at different scan rates. $213.7^{\circ} \mathrm{C}$. $\mathrm{TrxSO}_{2}$ showed on the second heating a glass transition at $119.3^{\circ} \mathrm{C}$ but no cold crystallization and only a tiny peak representing melting point indicating that the material is mostly amorphous. Additionally, powder diffraction patterns confirmed the crystalline nature of both as-synthesized truxenes (Fig. S8 $\dagger$ ) in their powder form. Thermogravimetric analysis proved the high thermal stability of $\mathbf{T r x S}$ with 5\% mass loss at the temperature of $326{ }^{\circ} \mathrm{C}$ and for $\mathbf{T r x S O}_{2} 357^{\circ} \mathrm{C}$ (Fig. S6 and S7†) which can be attributed to the all-aromatic backbone. Both DSC and TGA show how the introduction of oxygen atoms affects thermal properties where the melting point and glass transition temperature increase. Moreover, the sulfonyl group probably prevents stacking of the rings and leads to a more stable amorphous state. All these indicate that monothiatruxenes are promising candidates for optoelectronic applications.

In conclusion, we designed and synthesized monothiatruxene, its oxidized derivative and their brominated counterparts. The presented work shows that introduction of a sulfur atom to the truxene structure affects its electronic properties while maintaining high thermal stability. Additionally, due to the low symmetry of the molecule and presence of sulfur, a wider range of modifications is possible than that for truxene. Importantly, these materials also show high solubility.

\section{Author contributions}

The manuscript was written through contributions of all authors. All authors have given approval to the final version of the manuscript.

\section{Conflicts of interest}

There are no conflicts to declare.

\section{Acknowledgements}

We thank the International PhD Projects Programme of the Foundation for Polish Science, co-financed from European Regional Development Fund within Innovative Economy Operational Programme "Grants for innovation" (MPD-A1) and European Union's Horizon 2020 Research and Innovation Programme H2020-MSCA-IF-2014-659237 for financial support. We thank Krzysztof Górski for helpful discussions. 


\section{References}

1 W. Wislicenus, Ber. Dtsch. Chem. Ges., 1887, 20(1), 589.

2 F. Goubard and F. Dumur, RSC Adv., 2015, 5(5), 3521.

3 G. Zhang, F. Rominger and M. Mastalerz, Chem.-Eur. J., 2016, 22(9), 3084.

4 Y. Xie, X. Zhang, Y. Xiao, Y. Zhang, F. Zhou, J. Qi and J. Qu, Chem. Commun., 2012, 48(36), 4338.

5 X.-Y. Cao, X.-H. Liu, X.-H. Zhou, Y. Zhang, Y. Jiang, Y. Cao, Y.-X. Cui and J. Pei, J. Org. Chem., 2004, 69(18), 6050.

6 Z. Yang, B. Xu, J. He, L. Xue, Q. Guo, H. Xia and W. Tian, Org. Electron., 2009, 10(5), 954.

7 M. Kimura, S. Kuwano, Y. Sawaki, H. Fujikawa, K. Noda, Y. Taga and K. Takagi, J. Mater. Chem., 2005, 15(24), 2393.

8 Y. M. Sun, K. Xiao, Y. Q. Liu, J. L. Wang, J. Pei, G. Yu and D. B. Zhu, Adv. Funct. Mater., 2005, 15(5), 818.

9 X. R. Zhang, W. Chao, Y. T. Chuai, Y. Ma, R. Hao, D. C. Zou, Y. G. Wei and Y. Wang, Org. Lett., 2006, 8(12), 2563.

10 Z. Lu, C. Li, T. Fang, G. Li and Z. Bo, J. Mater. Chem. A, 2013, 1(26), 7657.

11 X. Zong, M. Liang, C. Fan, K. Tang, G. Li, Z. Sun and S. Xue, J. Phys. Chem. C, 2012, 116(20), 11241.
12 X. Qian, Y. Z. Zhu, J. Song, X. P. Gao and J. Y. Zheng, Org. Lett., 2013, 15(23), 6034.

13 G. Tsiminis, Y. Wang, P. E. Shaw, A. L. Kanibolotsky, I. F. Perepichka, M. D. Dawson, P. J. Skabara, G. A. Turnbull and I. D. W. Samuel, Appl. Phys. Lett., 2009, 94(24), 243304.

14 R. Cayuela, H. T. Nguyen, C. Destrade and A. M. Levelut, Mol. Cryst. Liq. Cryst., 1989, 177(1), 81.

15 Y. Kobori, T. Toyooka, H. Mazaki and Y. Satoh, US 5855971, 1996.

16 M. Maciejczyk, K. Górski and M. PietraszkiewiczPL407006, 2014.

17 S. Y. Hyun, Y. H. Yoon and J. H. Song, KR20140000611, 2014. 18 J. Clayden, Organolithiums: Selectivity for Synthesis, Pergamon, 2002.

19 V. Pandya, M. Jain, B. V. Chaugule, J. S. Patel, B. M. Parmar, J. K. Joshi and P. R. Patel, Synth. Commun., 2012, 42(4), 497. 20 M.-T. Kao, J.-H. Chen, Y.-Y. Chu, K.-P. Tseng, C.-H. Hsu, K.-T. Wong, C.-W. Chang, C.-P. Hsu and Y.-H. Liu, Org. Lett., 2011, 13(7), 1714. 